\title{
Fault Diagnosis and Fault Tolerant Control for Non-Gaussian Singular Time-Delayed Stochastic Distribution Systems
}

\author{
Lina Yao, ${ }^{1}$ Long Feng, ${ }^{1}$ and Bin Jiang ${ }^{2}$ \\ ${ }^{1}$ School of Electrical Engineering, Zhengzhou University, Zhengzhou 450001, China \\ ${ }^{2}$ Nanjing University of Aeronautics and Astronautics, Nanjing, Jiangsu 210016, China \\ Correspondence should be addressed to Lina Yao; michelle_lnxq@126.com
}

Received 12 November 2013; Accepted 21 January 2014; Published 27 February 2014

Academic Editor: Shuping He

Copyright (c) 2014 Lina Yao et al. This is an open access article distributed under the Creative Commons Attribution License, which permits unrestricted use, distribution, and reproduction in any medium, provided the original work is properly cited.

\begin{abstract}
New integrated fault diagnosis (FD) and fault tolerant control (FTC) algorithm for non-Gaussian singular time-delayed stochastic distribution control (SDC) system is proposed in the paper. Different from general SDC systems, in singular time-delayed SDC systems, the relationship between the weights and the control input is expressed by a singular time-delayed state space model, which largely increases the difficulty in the FD and FTC design. An iterative learning observer (ILO) is designed to carry out the fault estimation. The influence of the time delay term is eliminated in the process of the proof of the observation error stability. The fault may be constant, slow varying, or fast varying. Combined with the switching control theory, based on the estimated fault information, the fault tolerant controller can be designed to make the postfault probability density function (PDF) still track the given distribution. Simulations are given to show the effectiveness of the proposed integrated FD and FTC algorithms.
\end{abstract}

\section{Introduction}

Reliability and stability are of paramount importance for practical processes. Fault detection, diagnosis (FDD), and fault tolerant control (FTC) have long been regarded as an important and integrated part in control system design. The research of FDD and FTC has been carried out for almost thirty years, mainly aiming at the deterministic systems. However, in practical system, there are various stochastic disturbances such as sensor noises, stochastic disturbance, fault, or random variation of parameters. Thus, the system representation should be based on various stochastic models as the starting point of FDD and FTC. To enhance the reliability of the practical stochastic system, the research of FDD and FTC of stochastic systems has long been one of the important areas of control theory and application [14]. Usually, two kinds of approaches can be used to deal with the FDD problems of stochastic systems. The first group of methods is originated from the statistic theory, where the ratio of likelihood and Bayesian methods are used to estimate the abrupt changes of the states or parameters of the concerned systems. In this context, the FDD algorithms are obtained by using some numerical computation methods such as the Monte Carlo methods or the particle filtering methods [5-8]. As for the second group of methods, observer and filtering design theory are employed where the min-max optimization techniques have been applied to the estimation error system in order to guarantee some of the required performances $[9,10]$. Passive method and active method are used to deal with the FTC problem of stochastic systems. For the passive fault tolerant control of stochastic systems, the related literatures are mainly concerning the robust fault tolerant control of stochastic systems [11-13]. For the active fault tolerant control of stochastic systems, the fault estimation information is used to carry out the controller reconfiguration [14-16].

There are many systems in practice where the output concerned is the probability density function (PDF) of the system output, rather than the actual output values. For such a group of systems, the output that can be used for the feedback control is the measured output PDFs. Such types of stochastic systems are called stochastic distribution systems which were defined in [17], for instance, the paper evenness control, chemical polymerization process, grinding process fineness distribution control, and the flame distribution control. These kinds of system are described by the relationship between the 
input and the output PDF instead of the normal relationship between the input and the output. Stochastic distribution systems can not only represent Gaussian systems, but also represent non-Gaussian systems, making the assumption of being subject to Gaussian distribution be deleted. The aim of FDD in SDC systems is to use the measured input and output PDFs to obtain the information of the fault aim [18-20]. An observer based algorithm was previously developed in [21] to detect the fault in the non-Gaussian SDC system. For most stochastic distribution systems, the research results focus on the FDD of such systems and little attention is paid to the FTC of such systems. When the objective probability density function is known, the purpose of FTC of the stochastic distribution systems is to design a fault tolerant controller to make the postfault PDF still track the given distribution [14-16]. When the objective PDF is not known in advance, the purpose of FTC of stochastic distribution systems is to design a fault tolerant controller to make the uncertainty of the system output still be minimized. In this case, the concept of entropy is introduced to the stochastic distribution system and the performance index about the entropy is minimized considering the effect of the fault, leading to the minimum entropy fault tolerant control of stochastic distribution systems.

In the above-mentioned SDC systems, only dynamic links between the inputs and the weights are considered. However, in practice, some algebraic relations also exist between the input and the weights, leading to a singular state space model between the weights and the control input. Such systems are called singular stochastic distribution control systems. The final purpose of the grinding processes is to obtain ore pulp with certain fineness distribution by grinding the raw ore, which is a typical stochastic distribution system. The main control system of the grinding process, such as the amount of ore, amount of water, two times amount of water, sump level, cyclone feed concentration, pressure, and rate of flow, has direct influence on the probability density function of fineness distribution. Such control systems can be regarded as the input control variables which control the system distribution probability density function. It shows that a singular stochastic distribution control model between the probability density function and the multilevel control loops composed of the above-mentioned main control systems can be built. In practice, time delays may exist in system modelling, the data (or image) processing, and transmission phases [22]. This is true especially in chemical processes and paper making processes, where ignorance of time delays will make the related FDD and FTC approaches totally invalid. Thus, the singular stochastic distribution system model should include the time delay factor, leading to singular timedelayed stochastic distribution system model. Few literatures have been reported on the FDD and FTC of such singular time-delayed stochastic distribution system. This forms the main purpose of the work in this paper. In this context, an iterative learning (ILO) based fault diagnosis algorithm is proposed to estimate the change of fault. Using the fault estimation information and switching control theory, a fault tolerant controller is designed to make the postfault PDF still track the given distribution.
The rest of this paper is organized as follows. Section 2 presents model description. In Section 3, the iterative learning observer based fault diagnosis algorithm is proposed. Section 4 gives the design of the fault tolerant control. Simulation results of FD and FTC are presented in Section 5, followed by some concluding remarks in Section 6 .

\section{Model Description}

Denote $\gamma(y, u(t))$ as the probability density function of the system output $y$ being defined on a known bounded interval $[a, b]$. The model of the continuous singular time-delayed stochastic distribution system is given as follows:

$$
\begin{gathered}
E \dot{x}(t)=A x(t)+A_{d} x(t-\tau(t))+B u(t)+N F(t), \\
V(t)=D x(t), \\
\gamma(y, u(t))=C(y) V(t)+T(y),
\end{gathered}
$$

where $x \in R^{n}$ is the system state vector, $u(t) \in R^{m}$ is the system input, $V(t) \in R^{n-1}$ is the weight vector, $F \in R^{m}$ is the system fault vector, and $\tau(t)$ is the time delay term. $A \in R^{n \times n}$, $A_{d} \in R^{n \times n}, B \in R^{n \times m}, D \in R^{(n-1) \times n}, E \in R^{n \times n}$, and $N \in R^{n \times m}$ are system parameter matrices with $\operatorname{rank}(E)=q<n$ (i.e., $E$ is a singular matrix). $C(y), T(y)$ are decided by the selected basis functions.

Assumption 1. The system is regular; that is, $|s E-A| \neq 0$.

Assumption 2. The system has no impulse; that is, $\operatorname{rank} E=$ $\operatorname{deg}|s E-A|$.

If the above-mentioned assumption conditions are satisfied, there exist two nonsingular matrices $Q$ and $P$ such that

$$
\begin{gathered}
Q E P=\left[\begin{array}{cc}
I_{q} & 0 \\
0 & 0
\end{array}\right], \quad Q A P=\left[\begin{array}{cc}
A_{1} & 0 \\
0 & I_{n-q}
\end{array}\right], \\
Q A_{d} P=\left[\begin{array}{cc}
A_{d 1} & 0 \\
0 & I_{n-q}
\end{array}\right],
\end{gathered}
$$

where $Q, P \in R^{n \times n}, A_{1} \in R^{q \times q}, A_{d 1} \in R^{q \times q}$, and $I_{i}$ is an $i$-order identity matrix:

$$
\begin{gathered}
\mathrm{Q} B=\left[\begin{array}{l}
B_{1} \\
B_{2}
\end{array}\right] . \\
D P=\left[D_{1}, D_{2}\right], \quad D_{1} \in R^{(n-1) \times q}, D_{2} \in R^{(n-1) \times(n-q)} .
\end{gathered}
$$

By applying the following state coordinate transformation

$$
x(t)=P\left[\begin{array}{l}
x_{1}(t) \\
x_{2}(t)
\end{array}\right],
$$


where $x_{1}(t) \in R^{q \times 1}, x_{2}(t) \in R^{(n-q) \times 1}$, it can be obtained from (1) that

$$
\begin{gathered}
\dot{x}_{1}(t)=A_{1} x_{1}(t)+A_{d 1} x_{1}(t-\tau(t))+B_{1} u(t)+N_{1} F(t), \\
x_{2}(t)=-B_{2} u(t)-N_{2} F(t), \\
V(t)=D_{1} x_{1}(t)+D_{2} x_{2}(t), \\
\gamma(y, u(t))=C(y) V(t)+T(y) \\
=C(y)\left[D_{1} x_{1}(t)+D_{2} x_{2}(t)\right]+T(y) .
\end{gathered}
$$

\section{Fault Diagnosis}

The iterative learning observer based method is used to carry out fault diagnosis for the singular time-delayed stochastic distribution system, using the adaptive tuning property of the iterative learning observer. The fault diagnosis is constructed for the transformed system (5) as follows:

$$
\begin{gathered}
\dot{\hat{x}}_{1 m}(t)=A_{1} \widehat{x}_{1 m}(t)+B_{1} u(t)+A_{d} \widehat{x}_{1 m}(t-\tau(t)) \\
+L \xi_{m}+v(t), \\
\dot{\hat{x}}_{2 m}(t)=-B_{2} u(t), \\
v(t)=K_{1} v(t-g)+K_{2} \xi_{m}(t-g), \\
\dot{\widehat{F}}=W v(t), \\
\gamma_{m}(y, u(t))=C(y)\left[D_{1} \dot{\hat{x}}_{1 m}(t)+D_{2} \widehat{x}_{2 m}(t)\right]+T(y),
\end{gathered}
$$

where $\widehat{x}_{1 m}$ and $\widehat{x}_{2 m}$ are the state estimation, $g$ is the time delay of the ILO, $\xi_{m}(t)$ is the measured residual signal, $L$ and $K_{i}$ ( $i=$ $1,2)$ are the gain matrices, and $v(t)$ is the input signal of the ILO. From the third equation of the fault diagnosis observer, it can be seen that $v(t)$ is updated by the previous residual signal and the input information. The concept of "iterative" shows that the repeated operation can be carried out.

Denote the observation error vector as follows:

$$
e_{m}(t)=x_{1}(t)-\widehat{x}_{1 m}(t) .
$$

Then the residual signal can be formulated to give

$$
\begin{aligned}
\xi_{m}(t) & =\int_{a}^{b} \sigma(y)\left[\gamma(y, u(t))-\gamma_{m}(y, u(t))\right] d y \\
& =\int_{a}^{b} \sigma(y) C(y) d y\left[D_{1} x_{1}(t)-\widehat{x}_{1 m}(t)+D_{2} N_{2} F\right] \\
& =\Sigma D_{1} e_{m}(t)+\Sigma D_{2} N_{2} F,
\end{aligned}
$$

where $\Sigma=\int_{a}^{b} \sigma(y) C(y) d y$ and $\sigma(y)$ is the function of $y$ defined on $[a, b]$. If $\sigma(y)=1, \varepsilon_{m}$ is not used as $\gamma(y, u(t))$ and $\gamma_{m}(y, u(t))$ are integrated into 1 on $[a, b]$ simultaneously. From the transformed stochastic distribution system (5) and fault diagnosis observer (6), the observation error dynamic system can be obtained as

$$
\begin{aligned}
\dot{e}_{m}(t) & =\dot{x}_{1}(t)-\dot{\bar{x}}_{m}(t) \\
& =A_{1} e_{m}(t)+N_{1} F-L \xi_{m}-v(t)+A_{d} e_{m}(t-\tau(t)),
\end{aligned}
$$

where $G=N_{1}-L \Sigma D_{2} N_{2}$. It can be further formulated to give $\dot{e}_{m}(t)=\left(A_{1}-L \Sigma D_{1}\right) e_{m}(t)+G F-v(t)+A_{d} e_{m}(t-\tau(t))$.

It is assumed that $\left(A_{1}, \Sigma D_{1}\right)$ is observable.

Lemma 3. If the input of the iterative learning observer is defined by (6), the following inequality holds:

$$
\begin{aligned}
v^{T} v & \leq 3 v^{T}(t-g) K_{1}^{T} K_{1} v(t-g) \\
& +3 e_{m}^{T}(t-g)\left(K_{2} \Sigma D_{1}\right)^{T}\left(K_{2} \Sigma D_{1}\right) e_{m}(t-g) \\
& +3\left(K_{2} \Sigma D_{2} N_{2} F\right)^{T}\left(K_{2} \Sigma D_{2} N_{2} F\right) .
\end{aligned}
$$

Assumption 4. Assume that when $t \geq 0$, the input $u(t)$ and fault of the system are bounded:

$$
\|u\| \leq b_{u}, \quad\|F\| \leq f_{d} .
$$

Theorem 5. It is assumed that Assumption 4 is satisfied and there exist positive definite matrices $P_{1}, Q_{1}$, and $R_{1}$ to make the following equation hold:

$$
\left(A_{1}-L \Sigma D_{1}\right)^{T} P_{1}+P_{1}\left(A_{1}-L \Sigma D_{1}\right)+R_{1}+P_{1} P_{1}=-Q_{1} .
$$

Then the observation error system (10) is stable and observation error $e(t)$ is bounded.

Proof. The Lyapunov function is selected as follows:

$$
\begin{aligned}
\pi= & e_{m}^{T} P_{1} e_{m}+\int_{t-g}^{t} e_{m}^{T}(\theta) R_{1} e_{m}(\theta) d \theta \\
& +\int_{t-g}^{t} v^{T}(\alpha) v(\alpha) d \alpha+\eta \int_{t-\tau(t)}^{t} e_{m}^{T}(s) e_{m}(s) d s .
\end{aligned}
$$

The first order derivative of the Lyapunov function $\pi$ is derived as follows:

$$
\begin{aligned}
\dot{\pi} \leq & e_{m}^{T}\left(\left(A_{1}-L \Sigma D_{1}\right)^{T} P_{1}+P_{1}\left(A_{1}-L \Sigma D_{1}\right)+R_{1}+P_{1} P_{1}\right) e_{m} \\
& +2 f_{d}\left\|P_{1} G\right\|\left\|e_{m}\right\|+V^{T}(t-g) \\
& \times\left((6+3 \sigma)\left(K_{2} \Sigma D_{1}\right)^{T}\left(K_{2} \Sigma D_{1}\right)-R_{1}\right) e_{m}(t-g) \\
& -\sigma v^{T} v+(6+3 \sigma)\left(K_{2} \Sigma D_{2} N_{2} F\right)^{T}\left(K_{2} \Sigma D_{2} N_{2} F\right) \\
& +\frac{2 T_{2}\|V\|\left\|e_{m}\right\|\left\|P_{1} L \Sigma\right\|\|D\|\left\|e_{m}\right\|}{\sqrt{\|E\|}} .
\end{aligned}
$$


It is known that the following inequality holds: $2\left\|e_{m}^{T} P_{1}\right\|\|v(t)\| \leq e_{m}^{T} P_{1} P_{1} e_{m}+v^{T} v$.

It can be further obtained that

$$
\begin{aligned}
\dot{\pi} \leq & 2 f_{d} \lambda_{\max }\left(P_{1} G\right)\left\|e_{m}\right\| \\
& -\left(\lambda_{\min }\left(Q_{1}\right)-\frac{2 T_{2}\left\|P_{1} L \Sigma\right\|\|D\|}{\sqrt{\|E\|}}\right)\left\|e_{m}\right\|^{2} \\
& +(6+3 \sigma)\left(K_{2} \Sigma D_{2} N_{2} F\right)^{T}\left(K_{2} \Sigma D_{2} N_{2} F\right)-\sigma v^{T} v .
\end{aligned}
$$

When the condition $\lambda_{\min }\left(Q_{1}\right) \geq 2 T_{2}\left\|P_{1} L \Sigma\right\|\|D\| / \sqrt{\|E\|}$ is satisfied, the observation error system is asymptotically stable.

\section{Fault Tolerant Control}

When the fault is diagnosed, the fault tolerant control should be considered. Using the fault estimation information, the fault tolerant controller is designed to compensate the system performance loss caused by the fault. In this section, the performance index and the switching rules of the switched singular time delay system are introduced. When the fault happened, the performance index is minimized combining with the system model and the fault estimation information, leading to fault tolerant control of the singular time-delayed stochastic distribution system.

4.1. Design of the Faultless Controller. In this section, the purpose of the faultless PDF tracking controller is to choose a suitable control input to make the output probability density function track the given distribution. Different from the ordinary stochastic distribution system, $E$ before $\dot{x}$ is a singular matrix in the dynamic model.

The performance index of the switching time-delayed singular system is given as follows:

$$
\begin{aligned}
J_{1}(u(t)) & =\int_{a}^{b}\left(\gamma\left(y_{1}, u(t)\right)-g(y)\right)^{2} d y+u^{T}(t) R_{2} u(t) \\
& =\int_{0}^{+\infty}\left(x^{T}(t) Q_{2} x(t)+u_{\sigma(t)}^{T}(t) R_{2} u_{\sigma(t)}(t)\right) d t
\end{aligned}
$$

where $Q_{2}=Q_{2}^{T} \in R^{m \times m}>0$ and $R_{2}=R_{2}^{T} \in R^{m \times m}>0$ is the positive definite weight matrix of restricting the control input $u_{1}(t) \cdot u_{\sigma(t)}=G_{t} x(t)$ is the control rule of the switching time-delayed singular system.

It is further formulated to give that

$$
\begin{aligned}
J & =\int_{0}^{+\infty}\left(x^{T}(t) Q_{2} x(t)+u_{\sigma(t)}^{T} R_{2} u_{\sigma(t)}(t)\right) d t \\
& =\sum_{i=1}^{m} \sum_{j=1}^{\infty} \int_{t_{i j}}^{t_{i j+1}}\left(x^{T}(t) Q_{2} x(t)+u_{i}^{T}(t) R_{2} u_{i}(t)\right) d t
\end{aligned}
$$

$$
\begin{aligned}
& =\sum_{i=1}^{m} \sum_{j=1}^{\infty} \int_{t_{i} j}^{t_{i} j+1} x^{T}(t)\left(Q_{2}+G_{i}^{T} R_{2} G_{i}\right) x(t) d t \\
& <-\sum_{k=0}^{\infty} \int_{t_{k}}^{t_{k+1}} \dot{V}\left(x_{t}\right) d t \\
& =V\left(x\left(t_{0}\right)\right)=\Phi^{T}(0) P_{2}^{T} E \Phi(0)+\int_{-\tau}^{0} \Phi^{T}(s) Z \Phi(s) d s \\
& =\Phi_{1}^{T}(0) P_{2} \Phi_{1}(0)+\int_{-\tau}^{0} \Phi^{T}(s) Z \Phi(s) d s,
\end{aligned}
$$

where $Z \in R^{m \times m}$ is a symmetrically positive definite matrix.

From the above-mentioned inequality, it is known that

$$
J \leq \Phi_{1}^{T}(0) P_{2} \Phi_{1}(0)+\int_{-\tau}^{0} \Phi^{T}(s) Z \Phi(s) d s
$$

where $\Phi(t)$ is the continuous system initial function, $\Phi(t)=$ $\left[\Phi_{1}(t), \Phi_{2}(t)\right], \Phi_{1}(t) \in R^{r}, \Phi_{2}(t) \in R^{n-r}$, and $P_{2}=P_{2}^{T}>0$.

Denote $J^{*}$ as the upper bound of the performance function and it is shown that

$$
J^{*}=\Phi_{1}^{T}(0) P_{2} \Phi_{1}(0)+\int_{-\tau}^{0} \Phi^{T}(s) Z \Phi(s) d s
$$

Using the switching rule

$$
\begin{gathered}
\sigma(t)=\operatorname{argmin}\left\{x^{T}\left(\gamma(t)+P_{2}^{T} A_{\sigma(t)} Z^{-1} A_{\sigma(t)}^{T} P_{2}\right) x\right\}, \\
\gamma_{t}=P_{2}^{T} A_{\sigma(t)}+A_{\sigma(t)}^{T} P_{2}+Z+Q_{2}+G_{t}^{T} R_{2} G_{t} .
\end{gathered}
$$

The Lyapunov function is selected as follows:

$$
\begin{aligned}
V(t) & =X^{T}(t) P_{2}^{T} E x(t)+\int_{t-\tau}^{t} x^{T}(s) Z x(s) d s \\
& =\Phi_{1}^{T}(0) P_{2} \Phi_{1}(0)+\int_{-\tau}^{0} \Phi^{T}(s) Z \Phi(s) d s=J^{*} .
\end{aligned}
$$

The whole closed-loop system can be written as

$$
\begin{gathered}
E \dot{x}(t)=A_{\sigma(t)} x(t)+A_{\gamma \sigma(t)} x(t-\tau)+B_{\sigma(t)} u_{\sigma(t)}(t), \\
x(t)=\Phi(t), \quad t \in[-\tau, 0],
\end{gathered}
$$

where $A_{\gamma \sigma(t)}, A_{\sigma(t)}$, and $B_{\sigma(t)}$ are the constant matrices with appropriate dimensions under the designed switching rule $\sigma(t)$ of the system. 
The upper bound of the performance function is obtained as

$$
\begin{aligned}
J^{*}= & J_{1}(u(t))=\int_{a}^{b}(\bar{g}(y, t)-H(y) u(t))^{2} d y \\
& +u^{T}(t) R_{2} u(t)+\int_{-\tau}^{0} \Phi^{T}(s) Z \Phi(s) d s \\
= & \int_{a}^{b} \bar{g}^{2}(y, t) d y-\left[\int_{a}^{b} \bar{g}(y, t) \gamma(y) d y\right] u(t) \\
& -u^{T}(t) \int_{a}^{b} H^{T}(y) \bar{g}(y, t) d t \\
& +u^{T}(t) \int_{a}^{b} H^{T}(y) H(y) d y u(t) \\
& +u^{T}(t) R_{2} u(t)+\int_{-\tau}^{0} \Phi^{T}(s) Z \Phi(s) d s,
\end{aligned}
$$

where $s=\int_{a}^{b} H^{T}(y) H(y) d y, H(y)=C(y) D_{2} B_{2}$, and $\bar{g}(y, t)=C(y) D_{1} x_{1}(t)+T(y)-g(y)$.

Then the controller can be formulated to give

$$
\begin{aligned}
u(t)= & \left(s+R_{2}\right)^{-1} \int_{a}^{b} H^{T}(y) \bar{g}(y, t) d y \\
& -\int_{-\tau}^{0} \Phi^{T}(s) Z \Phi(s) d s .
\end{aligned}
$$

4.2. Design of the Fault Tolerant Controller after Fault Occurs. When the fault happens in the system, a new performance index function is given as

$$
\begin{aligned}
J_{2}\left(u^{\prime}(t)\right)= & \int_{a}^{b}\left(\gamma\left(y, u^{\prime}(t)\right)-g(y)\right)^{2} d y \\
& +u^{\prime}(t) R_{2} u^{\prime}(t)+\int_{-\tau}^{0} \Phi^{T}(s) Z \Phi(s) d s
\end{aligned}
$$

where $u^{\prime}(t)$ is the controller input after fault occurs. It can be obtained that

$$
\begin{aligned}
& \gamma\left(y, u^{\prime}(t)\right)-g(y) \\
&=C(y) D_{1} x_{1}(t)+C(y) D_{2} x_{2}(t) \\
& \quad+T(y)-g(y) \\
&=C(y) D_{1} x_{1}(t)+C(y) D_{2} N_{2} F(t) \\
&-C(y) D_{2} B_{2} u^{\prime}(t)+T(y)-g(y) \\
&= \breve{g}(y, t)-H(y) u^{\prime}(t),
\end{aligned}
$$

where $\breve{g}(y, t)=C(y) D_{1} \widehat{x}_{1}(t)+C(y) D_{2} N_{2} \widehat{F}(t)+T(y)-g(y)$.
The fault tolerant controller $u^{\prime}(t)$ can be obtained by solving the following optimization problem using the defined performance index and the system model:

$$
\begin{array}{lc}
\min _{x, w, z, a, M} a+\operatorname{tr}(M) \\
\text { s.t. }\left\{\begin{array}{c}
\sum_{t=1}^{m} a_{1}[*]<0 \\
{\left[\begin{array}{cc}
-a & \Phi_{1}^{T}(0) \\
\Phi_{1}(0) & -x_{1}
\end{array}\right]<0} \\
{\left[\begin{array}{cc}
-M & N^{\prime T} \\
N^{\prime} & -Z
\end{array}\right]<0,}
\end{array}\right.
\end{array}
$$

where $\int_{-\tau}^{0} \Phi(s) \Phi^{T}(s) d s=N^{\prime} N^{\prime T}, N^{\prime}$ and $M$ are symmetrical positive definite matrix:

$$
[*]=\left[\begin{array}{ccccc}
\Xi_{i} & x^{T} & x^{T} & \Psi_{i}^{T} & A_{\tau i} Z \\
x & -A & 0 & 0 & 0 \\
x & 0 & -Q^{-1} & 0 & 0 \\
\Psi_{i} & 0 & 0 & -R^{-1} & 0 \\
Z A_{\tau i}^{T} & 0 & 0 & 0 & -Z
\end{array}\right]<0
$$

$\Psi_{i}$ is a known parameter matrix.

It can be formulated that

$$
J^{*}=k \text { (constant) } \text {. }
$$

Similar to $u(t)$, the following fault tolerant controller can be obtained as

$$
\begin{aligned}
u^{\prime}(t)= & \left(s+R_{2}\right)^{-1} \int_{a}^{b} H^{T}(y) \breve{g}(y, t) d y \\
& +\left(s+R_{2}\right)^{-1} \int_{a}^{b} H^{T}(y) C(y) D_{1} d y \\
& +\left(s+R_{2}\right)^{-1} \int_{a}^{b} H^{T}(y) C(y) D_{2} N_{2} d y \widehat{F}(t) \\
& +\left(s+R_{2}\right)^{-1} \int_{a}^{b} H^{T}(y)[T(y)-g(y)] d y \\
& -\int_{-\tau}^{0} \Phi^{T}(s) Z^{-1} \Phi(s) d s .
\end{aligned}
$$

Remark 6. Based on the non-Gaussian singular stochastic distribution model of literature [16], time delay factor is considered in this paper. Similar to the fault diagnosis algorithm of literature [16], the iterative learning observer based fault diagnosis algorithm is used to carry out fault diagnosis for non-Gaussian time delay singular stochastic distribution system. Combined with the switch control theory, the corresponding fault tolerant control law is obtained.

\section{A Computer Simulation Example}

To demonstrate the effectiveness of the fault tolerant control algorithm, the output probability density function $\gamma(y, u(t))$ is given as

$$
\gamma(y, u(t))=\omega_{1} \phi_{1}(y)+\omega_{2} \phi_{2}(y)+\omega_{3} \phi_{3}(y) .
$$


The following B-spline basis functions are used to approximate $\gamma(y, u(t))$ :

$$
\begin{aligned}
\phi_{1}(y)= & \left(\frac{1}{6} y^{3}+\frac{3}{2} y^{2}+\frac{9}{2} y+\frac{9}{2}\right) f_{1} \\
& +\left(-\frac{1}{2} y^{3}-\frac{5}{2} y^{2}-\frac{7}{2} y-\frac{5}{6}\right) f_{2} \\
& +\left(\frac{1}{2} y^{3}+\frac{1}{2} y^{2}-\frac{1}{2} y+\frac{1}{6}\right) f_{3} \\
& +\left(-\frac{1}{6} y^{3}+\frac{1}{2} y^{2}-\frac{1}{2} y+\frac{1}{6}\right) f_{4}, \\
\phi_{2}(y)= & \left(\frac{1}{6} y^{3}+y^{2}+2 y+\frac{4}{3}\right) f_{2} \\
& +\left(-\frac{1}{2} y^{3}-y^{2}+\frac{2}{3}\right) f_{3} \\
& +\left(\frac{1}{2} y^{3}-y^{2}+\frac{2}{3}\right) f_{4} \\
& +\left(-\frac{1}{6} y^{3}+y^{2}-2 y+\frac{4}{3}\right) f_{5}, \\
& +\left(-\frac{1}{6} y^{3}+\frac{3}{2} y^{2}-\frac{9}{2} y+\frac{9}{2}\right) f_{6}, \\
& \left.+\left(-\frac{1}{2} y^{3}+\frac{1}{2} y^{2}+\frac{1}{2} y+\frac{1}{6}\right) y_{4} y_{3}+\frac{7}{2} y-\frac{5}{6}\right) f_{5} \\
\phi_{3}(y)= & \left(\frac{1}{6} y^{3}+\frac{1}{2} y^{2}+\frac{1}{2} y+\frac{1}{6}\right) f_{3} \\
& +(y)
\end{aligned}
$$

where $f_{i}(i=1,2,3,4,5,6)$ is defined as follows:

$$
f_{i}(y)= \begin{cases}1, & y \in[i-4, i-3], \\ 0, & \text { otherwise. }\end{cases}
$$

The static model of the probability density function is shown as

$$
\gamma(y, u(t))=C(y) V(t)+T(y) .
$$

The dynamic model of the singular time-delayed stochastic distribution system is given as

$$
\begin{gathered}
E \dot{x}(t)=A x(t)+A_{d} x(t-\tau(t))+B u(t)+N F(t), \\
V(t)=D x(t) .
\end{gathered}
$$

The main matrices of the dynamic model are given as

$$
\begin{gathered}
E=\left(\begin{array}{ccc}
1.0108 & 0.5794 & 5.7745 \\
3.8263 & -2.0132 & 13.7264 \\
-5.5036 & 6.8344 & -12.1285
\end{array}\right), \\
D=\left(\begin{array}{ccc}
0.4953 & 3.3627 & -0.7513 \\
0.7674 & 0.8753 & -0.4944
\end{array}\right), \\
A=\left(\begin{array}{ccc}
-1.5675 & -2.5243 & 0.5341 \\
0.4014 & 1.4281 & -9.1249 \\
1.9462 & -0.1041 & 4.8273
\end{array}\right),
\end{gathered}
$$

$$
\begin{gathered}
A_{d}=\left(\begin{array}{ccc}
-1.0054 & -2.7458 & 0 \\
1.5782 & 0.1461 & -4.8423 \\
0 & -0.5293 & 1.8249
\end{array}\right), \\
B=\left(\begin{array}{c}
-3.5044 \\
-0.3042 \\
-8.2882
\end{array}\right), \quad N=\left(\begin{array}{c}
2.2470 \\
6.4054 \\
-2.1299
\end{array}\right) .
\end{gathered}
$$

Select the following nonsingular transformation matrix:

$$
\begin{aligned}
P & =\left(\begin{array}{ccc}
0.2019 & -0.1422 & 0.4830 \\
0.1631 & 0.3211 & 0.2028 \\
0.1976 & 0.1455 & -0.1049
\end{array}\right), \\
Q & =\left(\begin{array}{ccc}
0.1020 & 0.1422 & -0.1690 \\
0.3428 & 0.1860 & 0.4515 \\
-0.4155 & 0.2784 & 0.1172
\end{array}\right)
\end{aligned}
$$

The dynamic model of the time-delayed singular stochastic distribution system can be transformed as

$$
\begin{gathered}
\dot{x}_{1}(t)=A_{1} x_{1}(t)+B_{1} u(t)+A_{d} \widehat{x}_{1 m}(t-\tau(t)) \\
+L \xi_{m}+V(t), \\
\dot{\hat{x}}_{2 m}(t)=-B_{2} u(t), \\
V(t)=K_{1} V(t-g)+K_{2} \xi_{m}(t-g), \\
\gamma_{m}(y, u(t))=C(y)\left[D_{1} x_{1}(t)+D_{2} x_{2}(t)\right]+T(y) .
\end{gathered}
$$

The following parameter matrices and vectors can be obtained as

$$
A_{1}=\left(\begin{array}{cc}
-0.5 & -0.25 \\
0.11 & -0.17
\end{array}\right), \quad A_{d}=\left(\begin{array}{cc}
-0.1 & 0 \\
0 & -0.5
\end{array}\right)
$$$$
B_{1}=\left(\begin{array}{c}
1 \\
-5
\end{array}\right), \quad B_{2}=0.4, \quad N_{2}=0.6, \quad N_{1}=\left(\begin{array}{c}
1.5 \\
1
\end{array}\right) \text {, }
$$$$
D_{1}=\left(\begin{array}{ll}
0.5 & 1.9 \\
0.2 & 0.1
\end{array}\right), \quad D_{2}=\left(\begin{array}{c}
1 \\
0.6
\end{array}\right),
$$$$
K_{1}=\left(\begin{array}{cc}
0.62 & 0.31 \\
-0.28 & 0.43
\end{array}\right), \quad K_{2}=\left(\begin{array}{c}
0.41 \\
0.36
\end{array}\right), \quad L=\left(\begin{array}{c}
0.5 \\
-0.6
\end{array}\right) \text {, }
$$$$
\Phi(t)=\left(\begin{array}{c}
\frac{1}{4} e^{t+1} \\
0
\end{array}\right), \quad t \in[-1,0],
$$

$$
\begin{aligned}
& \Phi(0)=\left(\begin{array}{c}
\frac{1}{4} e \\
0
\end{array}\right) \approx\left(\begin{array}{c}
0.6796 \\
0
\end{array}\right), \quad M=\left(\begin{array}{cc}
1.5680 & 0 \\
0 & 1.1459
\end{array}\right), \\
& \Psi_{1}=(-0.0362-0.3155), \quad \Psi_{2}=\left(\begin{array}{ll}
-0.0275 & -0.1961
\end{array}\right) .
\end{aligned}
$$

In this paper, the fault diagnosis and fault tolerant control algorithms can apply to abrupt fault and gradual fault. To 


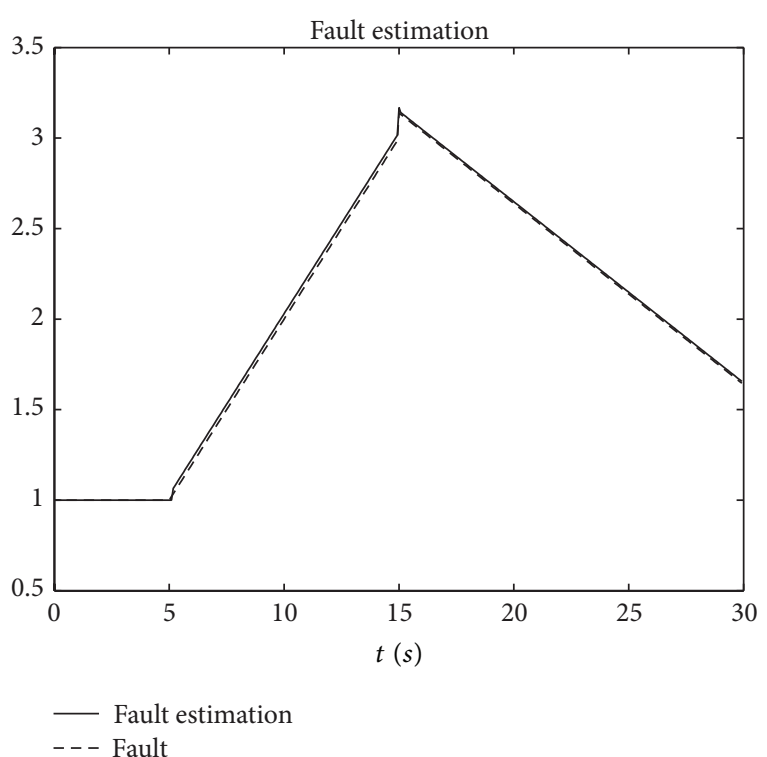

FIGURE 1: Fault estimation result.

System of three-dimensional graph (PDF)

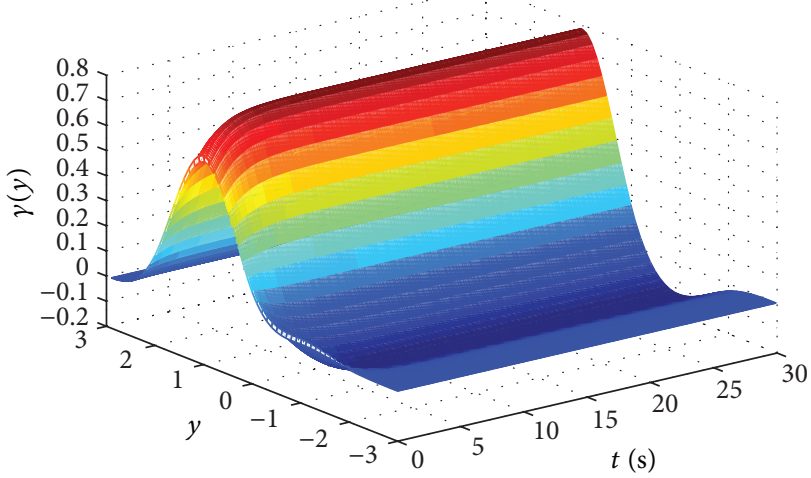

FIgure 2: The faultless PDF.

demonstrate the effectiveness of the algorithms, gradualvarying and abrupt fault are considered, respectively.

(1) A Gradual-Varying Fault. A gradual-varying fault is considered as follows:

$$
F= \begin{cases}0 & t<5 s \\ 0.4(t-5) & 5 s \leq t<15 s \\ 5.2-0.26(t-10.3) & t \geq 15 s\end{cases}
$$

where the learning rate $W$ is chosen as $W=\left(\begin{array}{c}33 \\ 15\end{array}\right)$. Figure 1 gives the fault estimation result. It can be seen that the tracking effect is good enough to show the effectiveness of the fault diagnosis algorithm. Figure 2 represents the faultless PDF and Figure 3 gives the postfault output probability density function. It is shown that fault tolerant control algorithm is effective to the fast-varying fault.

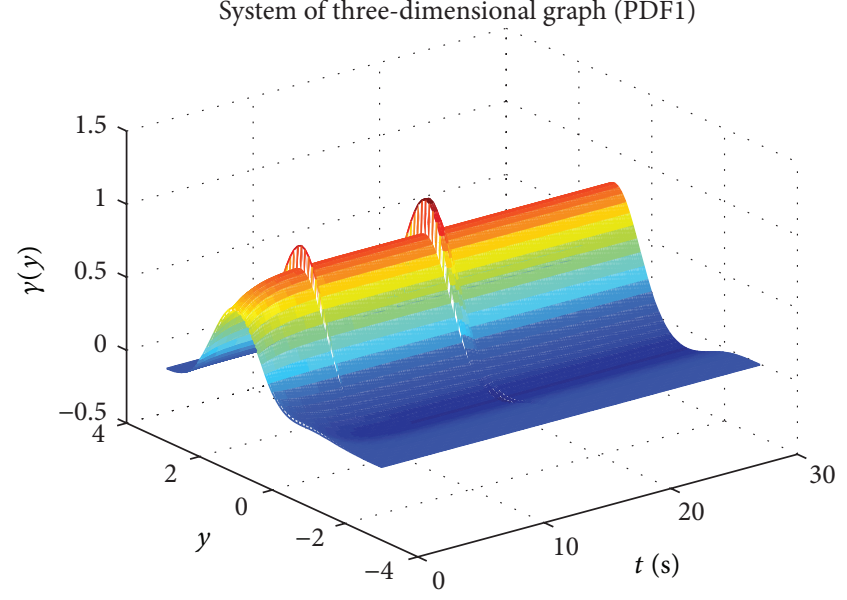

Figure 3: The postfault PDF.

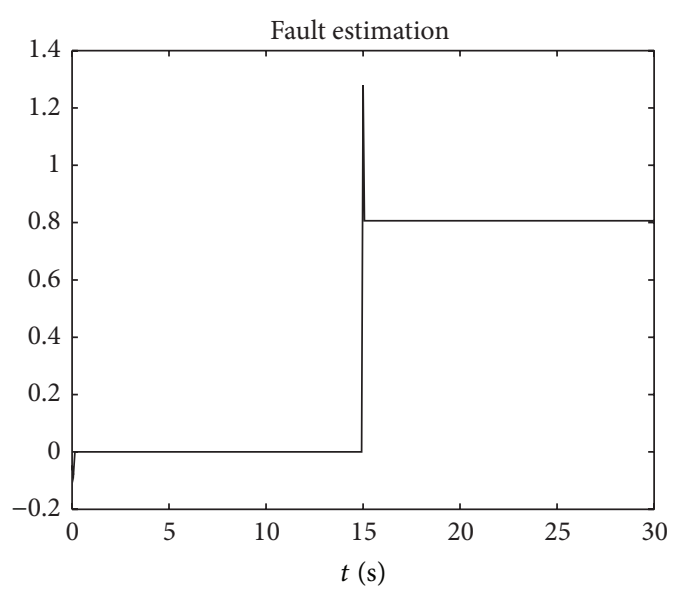

FIGURE 4: Fault estimation result.

(2) An Abrupt Fault. It is assumed that an abrupt fault is given as follows:

$$
F= \begin{cases}0 & t<15 s \\ 0.8 & t \geq 15 s\end{cases}
$$

The learning rate $W$ is chosen as $W=\left(\begin{array}{l}36 \\ 14\end{array}\right)$. Figure 4 gives the fault estimation result of the abrupt fault. Figure 5 gives the faultless output PDF and the postfault probability density function result is shown in Figure 6. The postfault PDF can still track the given distribution, leading to good fault tolerant control results to the abrupt fault.

\section{Conclusions}

For non-Gaussian singular stochastic distribution system, the time delay factor is considered. The iterative learning observer is used to carry out the fault diagnosis. The concept of switching system is introduced to the time delay singular stochastic distribution system. A new fault tolerant control algorithm is proposed combining switch with the time delay singular stochastic distribution system to make the postfault 


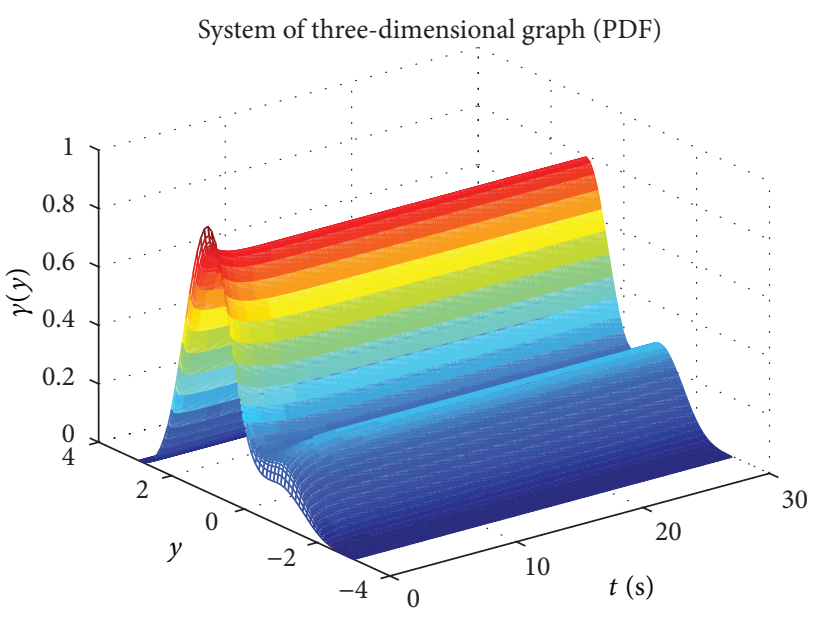

FIgURe 5: The faultless PDF.

System of three-dimensional graph (PDF)

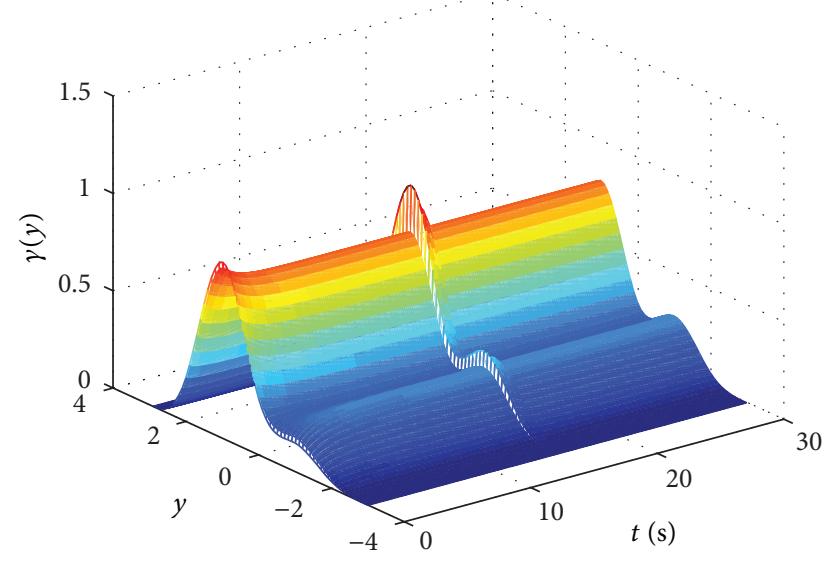

Figure 6: The postfault PDF.

probability density function still track the given distribution. The active fault tolerant control of the time delay singular stochastic distribution system is realized. The computer simulation results show that the fault diagnosis and fault tolerant control algorithms can apply not only to the abrupt fault, but also to the gradual-varying fault.

\section{Conflict of Interests}

The authors declare that there is no conflict of interests regarding the publication of this paper.

\section{Acknowledgments}

The authors would like to thank the financial support received from Chinese NSFC Grant 61374128, Research Fund for the Doctoral Program of Higher Education of China 20104101120007, and State Key Laboratory of Synthetical Automation for Process Industries.

\section{References}

[1] R. Isermann, "Model-based fault-detection and diagnosisstatus and applications," Annual Reviews in Control, vol. 29, no. 1, pp. 71-85, 2005.

[2] P. M. Frank and X. Ding, "Survey of robust residual generation and evaluation methods in observer-based fault detection systems," Journal of Process Control, vol. 7, no. 6, pp. 403-424, 1997.

[3] R. J. Patton and J. Chen, Control and Dynamic Systems: Robust Fault Detection and Isolation(FDI) Systems, Academic Press, London, UK, 1996.

[4] E. Martin, J. Morris, and S. Lane, "Monitoring process manufacturing performance," IEEE Control Systems Magazine, vol. 22, no. 5, pp. 26-39, 2002.

[5] M. Basseville, "On-board component fault detection and isolation using the statistical local approach," Automatica, vol. 34, no. 11, pp. 1391-1415, 1998.

[6] P. Li and V. Kadirkamanathan, "Particle filtering based likelihood ratio approach to fault diagnosis in nonlinear stochastic systems," IEEE Transactions on Systems, Man and Cybernetics C, vol. 31, no. 3, pp. 337-343, 2001.

[7] X. Wang and V. L. Syrmos, "Interacting multiple particle filters for fault diagnosis of non-linear stochastic systems," in Proceedings of the American Control Conference, pp. 4274-4279, June 2008.

[8] U. Münz and P. J. Zufiria, "Diagnosis of unknown parametric faults in non-linear stochastic dynamical systems," International Journal of Control, vol. 82, no. 4, pp. 603-619, 2009.

[9] R. H. Chen, D. L. Mingori, and J. L. Speyer, "Optimal stochastic fault detection filter," Automatica, vol. 39, no. 3, pp. 377-390, 2003.

[10] L. Q. Wu and D. W. C. Ho, "Fuzzy filter design for Itô stochastic systems with application to sensor fault detection," IEEE Transactions on Fuzzy Systems, vol. 17, no. 1, pp. 233-242, 2009.

[11] L. F. Ma, Z. D. Wang, Y. M. Bo, and Z. Guo, "Robust faulttolerant control for a class of nonlinear stochastic systems with variance constraints," Journal of Dynamic Systems, Measurement and Control, Transactions of the ASME, vol. 132, no. 4, pp. 1-6, 2010.

[12] Z. Lin, Y. Lin, and W. Zhang, "H stabilisation of nonlinear stochastic active fault-tolerant control systems: fuzzyinterpolation approach," IET Control Theory \& Applications, vol. 4, no. 10, pp. 2003-2017, 2010.

[13] M. Liu, X. B. Cao, and P. Shi, "Fault estimation and tolerant control for fuzzy stochastic systems," IEEE Transactions on Fuzzy Systems, vol. 21, no. 2, pp. 221-229, 2013.

[14] M. Liu and P. Shi, "Sensor fault estimation and tolerant control for Itô stochastic systems with a descriptor sliding mode approach," Automatica, vol. 49, no. 5, pp. 1242-1250, 2013.

[15] L. N. Yao, J. F. Qin, A. P. Wang, and H. Wang, "Fault diagnosis and fault-tolerant control for non-Gaussian non-linear stochastic systems using a rational square-root approximation model," IET Control Theory \& Applications, vol. 7, no. 1, pp. 116-124, 2013.

[16] L. Yao, J. Qin, H. Wang, and B. Jiang, "Design of new fault diagnosis and fault tolerant control scheme for non-Gaussian singular stochastic distribution systems," Automatica, vol. 48, no. 9, pp. 2305-2313, 2012.

[17] H. Wang, Bounded Dynamic Stochastic Systems: Modelling and Control, Springer, London, UK, 2000. 
[18] L. Guo and H. Wang, "Fault detection and diagnosis for general stochastic systems using B-spline expansions and nonlinear filters," IEEE Transactions on Circuits and Systems I, vol. 52, no. 8, pp. 1644-1652, 2005.

[19] H.-J. Ma and G.-H. Yang, "Observer-based fault diagnosis for a class of non-linear multiple input multiple output uncertain stochastic systems using B-spline expansions," IET Control Theory \& Applications, vol. 5, no. 1, pp. 173-187, 2011.

[20] Z. Gao, H. Wang, and T. Chai, "A robust fault detection filtering for stochastic distribution systems via descriptor estimator and parametric gain design," IET Control Theory \& Applications, vol. 1, no. 5, pp. 1286-1293, 2007.

[21] H. Wang and W. Lin, "Applying observer based FDI techniques to detect faults in dynamic and bounded stochastic distributions," International Journal of Control, vol. 73, no. 15, pp. 14241436, 2000.

[22] Q. X. Zhu, "Stabilization of stochastically singular nonlinear jump systems with unknown parameters and continuously distributed delays," International Journal of Control, Automation and Systems, vol. 11, no. 4, pp. 683-691, 2013. 


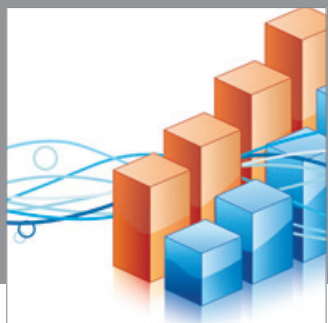

Advances in

Operations Research

mansans

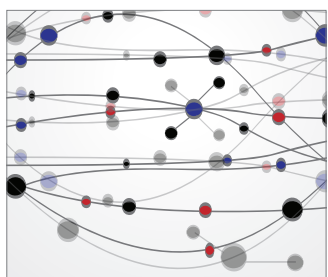

The Scientific World Journal
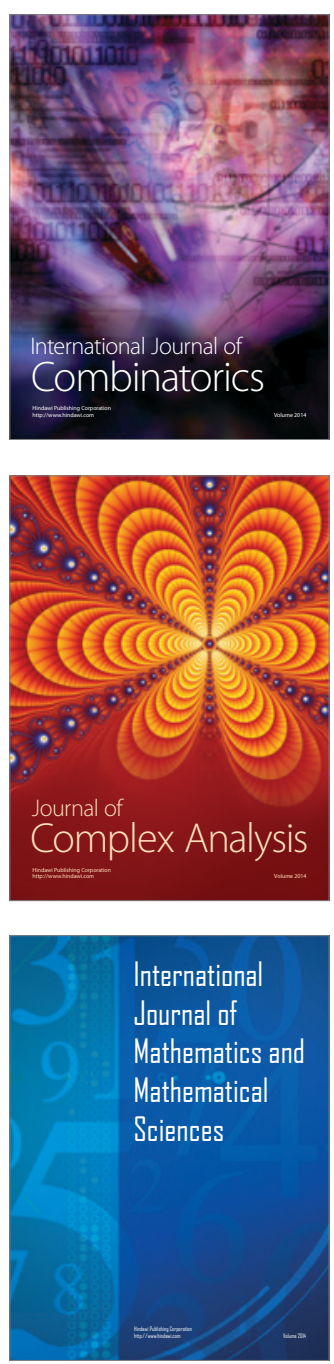
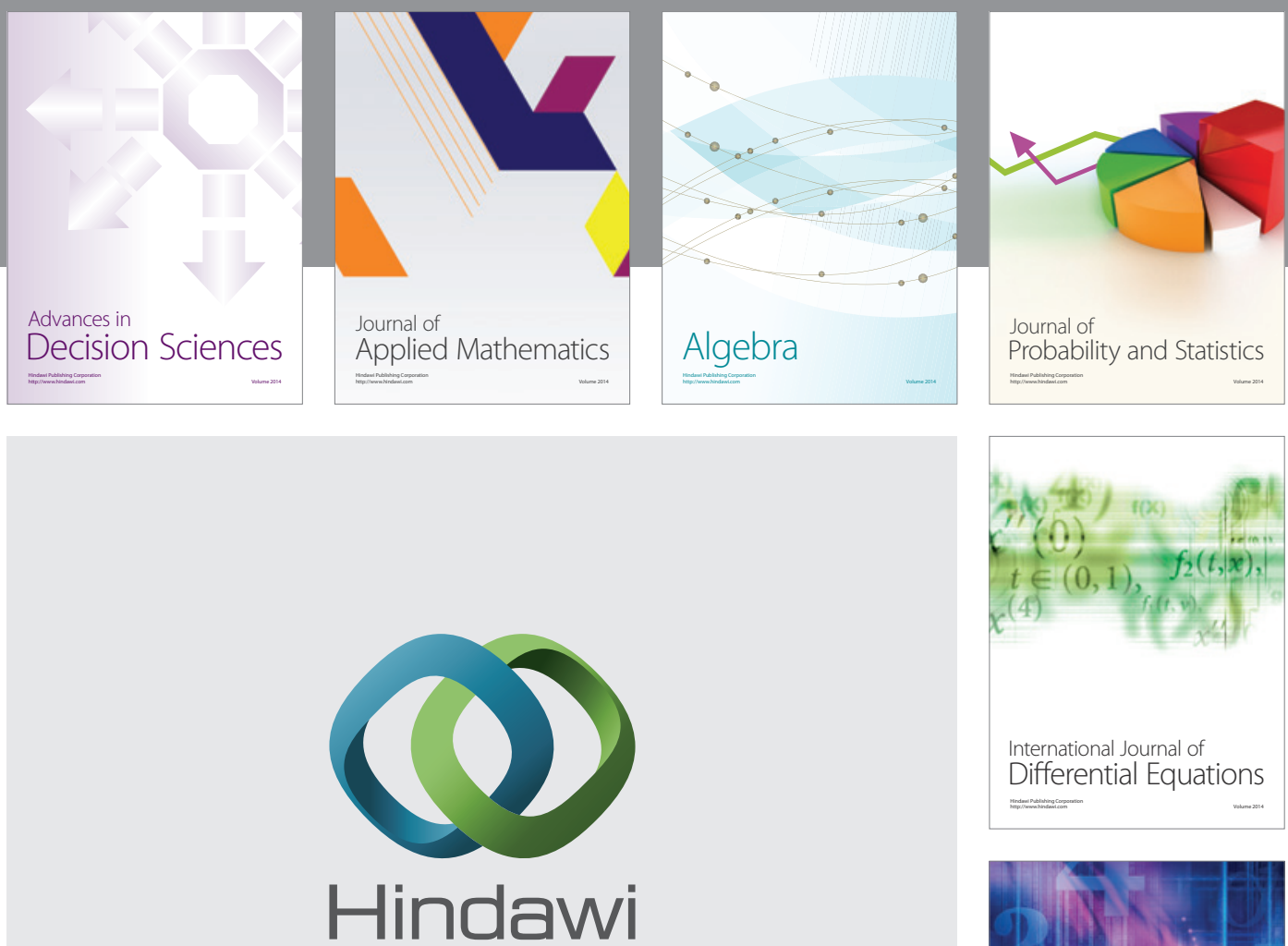

Submit your manuscripts at http://www.hindawi.com
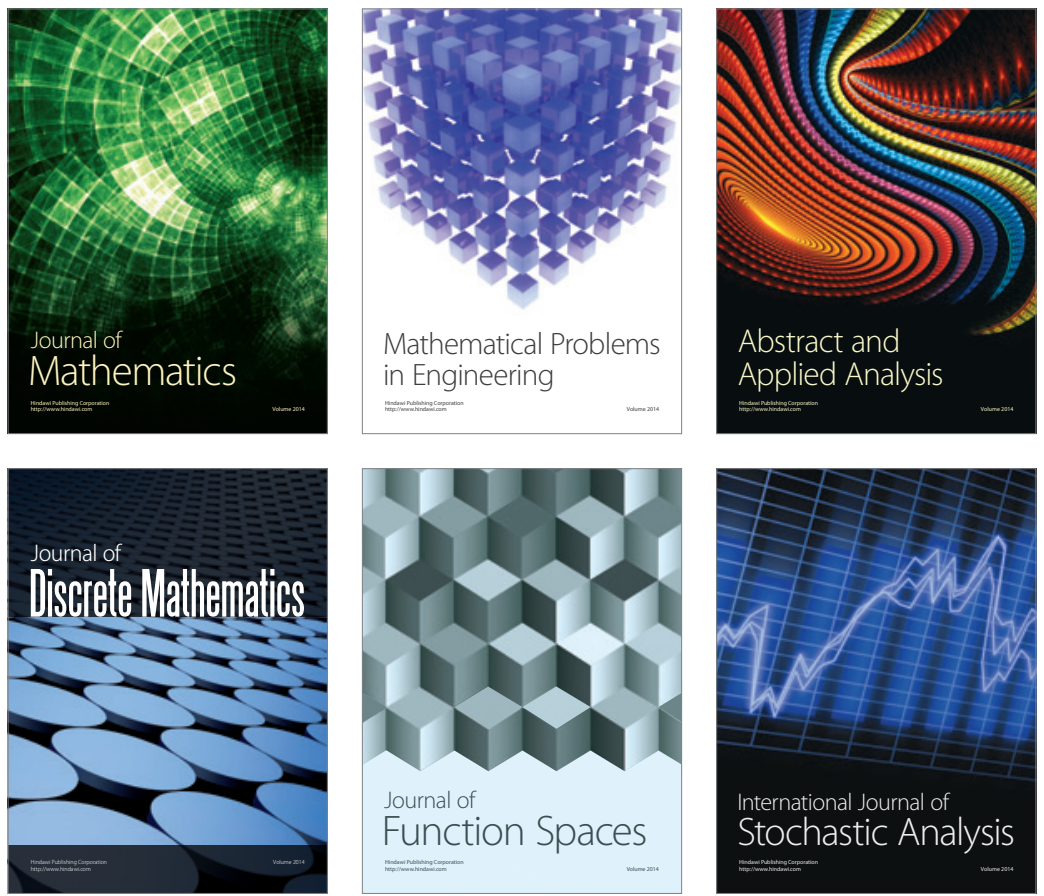

Journal of

Function Spaces

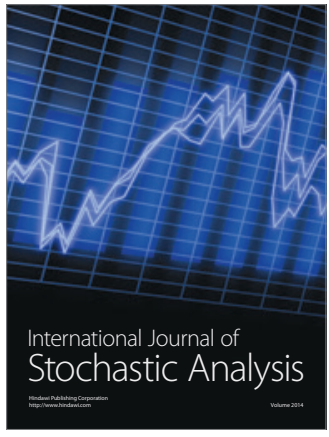

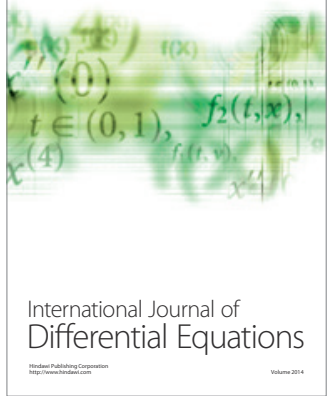
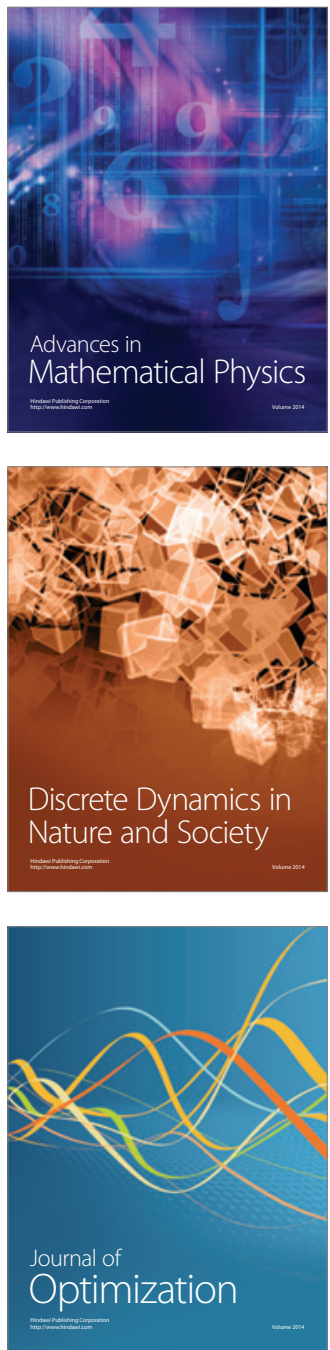